\title{
Editorial
}

\section{Quo Vadis Quasicrystals?}

\author{
Enrique Maciá Barber
}

Departamento de Física de Materiales, Facultad Ciencias Fisicas, Universidad Complutense de Madrid, E-28040 Madrid, Spain; emaciaba@fis.ucm.es; Tel.: +34-91-394-4745

Academic Editor: Helmut Cölfen

Received: 17 February 2017; Accepted: 22 February 2017; Published: 24 February 2017

\begin{abstract}
This Special Issue aims at gaining a deeper understanding on the relationship between the underlying structural order and the resulting physical properties in aperiodic systems, including quasicrystalline and related complex metallic alloys, photonic quasicrystals, and other structures exhibiting long-range aperiodic order. This Special Issue contains 12 papers which highlight recent developments in quasiperiodic crystal structure, photonic quasicrystals and related optical devices, the intrinsic electrical, thermal, and mechanical properties of icosahedral and decagonal metallic alloys, and the nature of chemical bonding in intermetallic compounds, from a multidisciplinary perspective. In light of the results presented in the contributions collected in this Special Issue, we can confidently expect that new insights into the interdisciplinary science of quasicrystals will be gained in the years to come, providing a sharper picture of their structures and related physical properties, and spurring further progress in practical issues related to both materials engineering science and nanotechnology.
\end{abstract}

Keywords: quasicrystals; aperiodic crystals; photonic quasicrystals; aperiodic structures; complex intermetallics; Hume-Rothery compounds; push-pull alloys; skutterudites; chemical bonding

\section{Quasiperiodic Crystals Structure}

The existence of a novel phase of matter, characterized by the presence of atomic long-range ordering along with icosahedral point group symmetry, was first reported on 12 November 1984 [1]. Six weeks later, the nature of this long-range order was explained by invoking the mathematical notion of quasiperiodic functions, thereby widening the concept of a periodic distribution of atoms through space to that of a quasiperiodic one [2]. In this way, the discovered novel phase was envisioned as a natural extension of the classical crystal notion, now embodying quasiperiodic arrangements of matter as well. Accordingly, the shorthand term quasicrystal (standing for quasiperiodic crystal) was coined. Daniel Shechtman was awarded the Nobel Prize in Chemistry in 2011 for "the discovery of quasicrystals". Dov Levine and Paul J. Steinhardt were awarded the 2010 Oliver Buckley Condensed Matter Prize from the American Physical Society for "pioneering contributions to the theory of quasicrystals, including the prediction of their diffraction pattern".

However, in retrospect these semantics may appear somewhat misleading since the prefix "quasi" apparently suggests that these materials occupy an intermediate position between well ordered arrangements of matter and disordered ones, hence denoting some sort of defective, glassy solids. On the contrary, it is now well established that thermodynamically stable quasicrystals (QCs) show long-range orderings of very high quality, rendering discrete diffraction patterns with a dense distribution of sharp Bragg peaks which can be properly indexed in terms of a suitably generalized crystallography [3,4]. Indeed, in 1992 the International Union of Crystallography widened the very definition of crystal, introducing two broad classes of crystalline representatives referred to as periodic and aperiodic crystals, respectively. According to the proposed terms of reference, by "crystal" we now mean any solid having an essentially discrete diffraction diagram, and by "aperiodic crystal" we 
mean any crystal in which three-dimensional lattice periodicity can be considered absent [5]. Thus, QCs along with incommensurate modulate phases and incommensurate composites, belong to the new aperiodic crystal category, whereas periodic crystals can be referred to as classical crystals.

Chronologically, these developments in crystallography resulted from the study not of newly discovered minerals but of synthetic alloys produced under controlled conditions in the laboratory. Quite remarkably, in 2009 the first mineral with an icosahedral quasicrystalline structure, named icosahedrite, was found within a meteorite collected from Khatyrka (eastern Russia), and six years later a new natural quasicrystal with decagonal symmetry (named decagonite) was also identified in the same meteorite [6-9]. Although mineral QCs naturally formed on Earth have not been reported to date, it is quite conceivable that some of these minerals may exist on our planet. In fact, synthesis experiments and crystallochemical analyses suggest that a number of minerals could be transformed into QCs under extreme pressure and temperature conditions on Earth [10-12]. Hence, finding such mineral QCs of terrestrial origin is relevant to the investigation of the formation conditions of aperiodic crystals in nature and would constitute a new contribution coming from the mineral kindom to the advancement of crystallography. This appealing issue is addressed in the contribution from Carlos M. Pina and Victoria Sánchez-Acevedo who, after summarizing a number of studies of known minerals with aperiodic crystal structures, discuss current investigations aimed at the search for new possible quasicrystalline minerals in nature [13]. Quite interestingly, among the proposed potential candidates are skutterudite compounds, which have been intensively considered in the quest for novel thermoelectric materials during the past two decades [14].

The 1992 revamped crystal definition reflects our current understanding that periodicity at the atomic scale is a sufficient but not necessary condition for crystallinity. Instead, the presence of a long-range atomic order rendering discrete diffraction patterns should be regarded as the essential attribute of crystalline matter rather than mere periodicity. Two long standing open questions in the field concern what distributions of atoms in space are able to diffract and how to determine the exact positions of these atoms from the obtained diffraction patterns and electron microscope images. In their contribution, Ruitao Li, Zhong Li, Zhili Dong, and Khiam Aik Khor review recent progress in the structural study of QCs using transmission electron microscopy, the same tool originally used to discover them, reporting on several findings regarding the symmetry, local atomic arrangement, and chemical order of both icosahedral and decagonal QCs [15]. Notwithstanding the progresses achieved, it is still difficult to obtain complete structural information about QCs by experimental techniques involving diffraction and imaging alone. Indeed, diffraction analysis can only provide the globally averaged structural information, which may differ considerably from the local details [16]. Thus, a combination of modelling and experiments seems to be essential to satisfactorily solve the structure of different QCs families.

Two main methods have been employed to geometrically model quasicrystalline structures, namely, high dimensional cut-and-project methods and geometric space tiling [17]. The proposed tiling models provide prescriptions for possible atomic configurations at both the local and global levels, including the atomic arrangement of clusters at different spatial scales. On the other hand, the recourse to a higher dimensional space-based crystallography allows one to recover the very possibility of describing the order present in aperiodic crystals in terms of a periodic distribution of hyperatoms located in a hyperspace which embeds the three-dimensional physical space. In this context, the contribution by Radoslaw Strzalka, Irineusz Buganski, and Janusz Wolny introduces an alternative approach which is based on the average unit cell concept: a probability distribution of atomic positions with respect to a suitable reference lattice in physical space [18]. This distribution carries the complete structural information required for structure determination via diffraction experiments regardless of the inner symmetry of the diffracting medium. In addition, these atomic probability distributions are sensitive to any disorder with respect to the ideal structure introduced by phonons and phasons as well. 
Although calculations performed within the statistical method framework are equivalent to those obtained from multidimensional analysis, and they avoid some drawbacks related to the physical interpretation of the so-called atomic surfaces (a basic concept in the higher dimensional approach), it is also true that the higher dimensional approach restores all hidden symmetries of QCs into the unit-cell of the hyperlattice. According to this view, a more appropriate term for these solids would probably be hypercrystals [19-21]. By adopting the hyperspace perspective, the contribution by Farhad A. Namin and Douglas H. Werner exploits the unit cell in the higher dimensional space to obtain the atomic surfaces' Fourier series expansion in closed-form [22]. This approach will eventually allow for a possible generalization from vertex models, which assume identical particles at all vertices, to a more realistic case where the $\mathrm{QC}$ is composed of different atoms. The method proposed to obtain the diffraction pattern can also be used to determine the photonic resonances related to the QC structure, thereby providing an interesting link between the diffraction methods and optical applications.

\section{Photonic Quasicrystals}

Light transmission through aperiodic media has garnered ever-increasing attention in order to understand the interplay between optical properties and the underlying aperiodic order of the substrate. To this end, the mathematical analogy between the Schrödinger equation describing the motion of an electron under the action of a potential and the Helmholtz equation describing a monochromatic electromagnetic wave propagating in a medium with a variable refractive index profile provides a helpful analogy involving basic concepts in modern optoelectonics [23]. This analogy, which borrows some basic notions such as Bloch waves or Brillouin zones from solid state physics, has been developed and successfully applied to numerous optical devices, which are of common use in current optoelectronics and optical communication applications.

In this way, the so-called photonic crystal concept has been introduced to describe optical systems which exhibit large frequency stop bands due to interference effects, in close analogy with the presence of band structures in conventional atomic lattices, or the formation of energy minibands in superlattices $[24,25]$. Quite interestingly, the very notion of photonic crystals can be extended to describe the properties of quasiperiodic photonic structures as well. To this end, one simply considers that the optical properties of the medium are given by a quasiperiodic refraction index function instead of a periodic one. The resulting structure can then be properly regarded as a photonic quasicrystal (PQC).

Long-range quasiperiodic order, on its own, endows PQCs with certain characteristic properties which are not exhibited by their periodic counterparts. For instance, due to their highly fragmented frequency spectrum, aperiodic multilayers offer more full transmission peaks than periodic ones in a given frequency range for a given system length, whereas the inflation symmetry gives rise to a denser Fourier spectrum structure. In addition, the existence of higher order rotational axes, including ten-fold or twelve-fold symmetries, and even larger ones approaching the "Bragg-ring" limit in the case of spiral lattices, leads to more isotropic complete photonic band gaps in PQCs, generally requiring lower refractive index contrast values than those observed for periodic photonic crystals [26,27].

Accordingly, some fundamental questions naturally arise regarding what can be done with photonic devices based on aperiodic arrangements that cannot be achieved with periodic based ones, or also concerning what the advantages of aperiodic optical systems are over both periodic and random ones. Some answers to these appealing issues are given in the contribution by Luca Dal Negro, Ren Wang, and Felipe A. Pinhero who provide a comprehensive discussion of the relationship between the structural and spectral properties that govern the distinctive optical behaviour in a number of representative systems exhibiting periodic, random, or quasi-periodic order, as well as spiral arrangements with a tunable degree of aperiodic order [28]. To this end, they introduce spectral graph theory concepts in order to perform spatial point pattern analyses along with a rigorous Green's matrix method, which provides access to the electromagnetic scattering behaviour of deterministic aperiodic optical media for the first time. 
Another important feature stemming from the underlying structural order in PQCs is the existence of the so-called critical modes, which are spatially more complex than those related to both extended Bloch and exponentially localized defect modes in periodic photonic crystals. The presence of these critical states gives rise to the possible existence of either relatively localized or significantly extended electromagnetic intensity patterns depending on the involved scattering processes. The occurrence of highly localized optical modes in the absence of disorder, that is, in defect-free PQCs, is of interest for the fabrication of high quality factor resonators as well as coupled-resonator waveguides. In fact, the presence of critically localized states generally offers a higher degree of design and tuning flexibility. Therefore, compared with periodic arrays, where the resonance of a nanocavity can be achieved for a single frequency with a specific electric field pattern, in aperiodic multilayers a multifrequency nanocavity array can exist, and for each frequency the electric field pattern has a different spatial behaviour. Thus, in contrast to periodic systems, spatial quasiperiodic order naturally introduces a full set of non-equivalent sites in 2D and 3D aperiodic arrangements. In this way, a great diversity of electromagnetic spatial patterns can be obtained for different frequencies. This property paves the way for the design of novel optical devices, as it is conveniently illustrated in the contribution from Gianluigi Zito, Giulia Rusciano, Antonio Sasso, and Sergio De Nicola who numerically investigate the electromagnetic field localization in a two-dimensional photonic structure generated with holographic tiling obtained by placing dielectric pillars at the maxima of a multi-beam interference pattern hologram [29]. This structure is inspired by the Ammann-Beenker QC, albeit lacking classically forbidden symmetries, and hence it belongs to a class of QCs which have spurred an increasing attention during the last decade [30,31]. Their study demonstrates that light confinement can be induced into an air mirrorless cavity by the inherent symmetry of the spatial distribution of the dielectric scatterers forming the side walls of the open cavity. Furthermore, the propagation direction can be controlled by suitable aperiodic designs of the structure, thereby introducing a new avenue for the design of photonic devices.

\section{Intrinsic Properties of Stable Quasicrystals}

The discovery of the first thermodynamically stable QC, exhibiting the growth morphology of a triacontahedron, was reported in 1986 in the Al-Cu-Li alloy system [32]. Shortly after, a second stable QC, exhibiting a pentagonal dodecahedron solidification morphology, was reported in the Ga-Mg-Zn system [33]. However, these QCs did not show good enough structural quality to properly ascertain the possible existence of physical properties directly related to their quasiperiodic arrangement of atoms throughout the space. Fortunately, a series of thermodynamically stable quasicrystalline alloys of high structural quality in the icosahedral $\mathrm{Al}-\mathrm{Cu}-(\mathrm{Fe}, \mathrm{Ru}, \mathrm{Os})$ [34], $\mathrm{Al}-\mathrm{Pd}-(\mathrm{Mn}, \mathrm{Re})$ [35], and $\mathrm{Zn}-\mathrm{Mg}-\mathrm{RE}$ systems [36], and the decagonal $\mathrm{Al}-\mathrm{Co}-(\mathrm{Cu}, \mathrm{Ni})$ system [37,38], were discovered during the period from 1987-1994. Titanium based stable icosahedral QCs in the Ti-Zr-Ni alloy system were also reported in 1994 [39], and stable QCs belonging to the Zn-Sc-RE, Cd-Mg-RE, or (Au,Ag)-In-Ca systems have been announced during the last decade [40]. The first stable binary QC was discovered in 2000 in the $\mathrm{Cd}-\mathrm{Yb}(\mathrm{Ca})$ system [41]. No stable single-component $\mathrm{QC}$ has been reported to date. In consideration of the key role played in most of these findings, An Pang Tsai was given the Jean Marie Dubois Award in 2005 "in recognition of fundamentally important discoveries of new quasicrystalline phases".

Currently, more than sixty different compounds, belonging to about twenty different alloy systems, have been reported to be thermodynamically stable up to their respective melting points and to exhibit Bragg peaks of extraordinary quality, comparable to those observed for the best monocrystalline samples ever grown [40]. The discovery of this set of large, high quality samples has allowed for the study of intrinsic physical properties of QCs, including detailed experimental studies of their related mechanical, electrical, thermal, and optical transport properties.

In light of the experimental knowledge gained during the last twenty five years on the intrinsic properties of stable QCs, it is now clear that they exhibit a remarkable number of unique physical properties [20,42,43], which significantly differ from those observed in their periodic counterparts. 
In fact, quasicrystalline alloys remarkably depart from standard metallic behaviour. In particular, the electrical transport properties of thermodynamically stable QCs of high structural quality resemble a more semiconductor-like than metallic character. Therefore, QCs provide an intriguing example of ordered solids made of typical metallic atoms which do not exhibit most of the physical properties usually signaling the presence of metallic bonding.

Are these new properties uniquely related to the quasiperiodic order present in the underlying structure? Previously, it was tacitly assumed that these specific properties should be somehow related to the new kind of order present in QCs, so that they could be regarded as the fingerprints of the quasiperiodic $(\mathrm{QP})$ order in matter. This rationale was additionally supported by the observation of remarkable anisotropies in the transport properties of decagonal QCs, which behave as metals when measurements are performed along their periodic direction, whereas they exhibit a non-metallic behaviour in the (quasiperiodically ordered) perpendicular planes. Nevertheless, to the best of my knowledge, no definitive theoretical explanation has yet been provided about the physical origin of the specific properties of QCs on the sole basis of the very nature of QP order itself.

By following a systematic research program considering the temperature dependence of specific heat along with the different transport properties (electrical conductivity, magnetic susceptibility, and Hall coefficient) of icosahedral AlCuFe samples over a broad temperature range, Alexander Prekul and Natalya Shchegolikhina propose in their contribution an electronic structure model which considers the presence of a couple of two-level localized electronic states superimposed onto a broad pseudogap [44]. The suitability of this model is substantiated by means of a fitting analysis of tunnel conductance spectra along with the analysis of optical conductivity spectra, clearly supporting the existence of several excitation energy scales in the considered samples. This work is probably unique in its goal of using a wide variety of experimental techniques as suitable diagnostic tools in order to grasp a deeper understanding of both the electronic structure and the underlying transport mechanisms of icosahedral QCs in a unified way, properly disclosing the existence of (at least) two relevant energy scales to be considered in the electronic structure. A detailed study of the Seebeck coefficient temperature dependence curves of these QCs during the next few years would be very appealing, in order to gain additional insights on the role of these fine electronic structural features in their transport properties.

The notion of phason is one of the most characteristic concepts we have inherited from the high dimensional crystallography approach and it is related to many physical effects in QCs, affecting their specific heat, lattice thermal conductivity, dislocation motion, atomic diffusion processes, and mechanical properties [42]. In their contribution, $\mathrm{Wu} \mathrm{Li}$ and Tianyou Fan review the elastic properties of QCs, particularly focusing on the role of phason elastic degrees of freedom and the coupling between phonon and phason elementary excitations in the dynamic deformation process [45]. By adopting a systematic tutorial approach, a variety of fundamental problems such as screw dislocation motion and crack propagation are described and properly illustrated with suitable application examples including decagonal, dodecagonal, and icosahedral QCs. Along the same vein, Mahmoud Hosseini, Jan Sladek, and Vladimir Sladek discuss the dynamical response of a cylinder made of materials with decagonal QC properties by considering both phonon and phason stress fields [46]. In this contribution, special attention is paid to the coupling between both fields in terms of waves propagating with a finite speed, making use of a methodology which can be easily extended to QCs exhibiting other structural symmetries.

\section{On the Nature of Chemical Bonding}

A growing number of experimental and numerical simulation evidences now suggest that a main factor determining the remarkable properties of both decagonal and icosahedral QCs is related to physicochemical aspects involving the nearest and next-nearest atomic neighbours, thereby highlighting the important role of chemical bonding in the emergence of the unusual physical properties of QCs [47-50]. In fact, whenever there are wave functions overlapping among contiguous atoms in a solid lattice (a concept closely related to that of chemical bonding), the possibility of charge 
delocalization (i.e., extended character of the electrons) naturally emerges. In turn, the electronic itineracy in a long-range ordered potential is at the basis of the Brillouin zone concept in solid state physics, which arises from diffraction and interference processes of electronic wave functions with arrangements of atomic planes (either periodically or quasiperiodically stacked). Indeed, it is the long-range order (not exclusively periodic one) which allows for a truly extended nature of charge carriers throughout a solid via resonant band formation. Accordingly, it seems reasonable to expect that the very nature of chemical bonding in QCs could play a significant role in the onset of most of their distinctive physical properties. Thus, rather than trying to explain the specific properties of QCs in terms of the conceptual schemes originally introduced to describe classical periodic solids (e.g., in terms of metallic, semimetallic, semiconductor, or insulator behaviours), it may be advisable to introduce broader perspectives able to properly account for the main features appearing in their electronic structure.

In their contribution, Uichiro Mizutani and Hirokazu Sato provide a detailed description of the different methods that have been employed to calculate a magnitude representing the number of itinerant electrons (the so-called electron per atom ratio, $e / a$ ), showing that it plays a key role in the bonding of a solid and is closely linked with the valence concept chemists frequently use [51]. To this end, the authors present a detailed update, written in a nice tutorial style, of the so-called full-potential linearized augmented plane wave (FLAPW)-Fourier theory [52]. This powerful approach analyzes the interference of itinerant electrons with appropriate sets of lattice planes, providing an efficient way to determine reliable $e / a$ values for 54 elements in the periodic table (including representatives from group 1 up to group 16) as well as that of several pseudogap-bearing binary and ternary intermetallic compounds, including $\mathrm{Al}-, \mathrm{Zn}-$, and Cd-based compounds, Zintl polar compounds, phosphorus-based compounds, and inter-transition metal compounds including Laves phases. These compounds are thus classified into several families on the van Arkel-Ketelaar triangle map according to their bonding-types. In so doing, U. Mizutani and H. Sato provide a deeper insight on the physical basis of the Hume-Rothery electron concentration rule in transition metal based compounds, including most relevant quasicrystalline forming alloys. Quite remarkably, the origin of a pseudogap at the Fermi level for a large number of materials is successfully interpreted in terms of interference conditions, regardless of the bond-types involved. It is also interesting to note that phosphorus bearing skutterudites (one of the compounds considered as a potential natural QC candidate in [13]) properly obey the Hume-Rothery electron concentration rule.

An interesting class of quasicrystal forming materials is found among the so-called "push-pull" alloys, a class of ternary compounds whose atomic constituents, say, A, B, and C, are chosen in such a way that B-C interactions are repulsive or very small, while A-B and A-C interactions are strongly attractive in the respective binary systems. The resulting alloys form complex intermetallics with tens to thousands of atoms in their unit cell, and a few of them lead to the ultimate degree of structural complexity, when quasiperiodic order substitutes for crystal periodicity. In their contribution, Esther Belin-Ferré, Guy Trambly de Laissardière, and Jean-Marie Dubois study the electronic structure of several push-pull alloys belonging to the ternary system $\mathrm{Al}-\mathrm{Cu}-\mathrm{Fe}$, of interest in quasicrystals research [53]. From their analysis, application-oriented properties such as high catalytic activity and infrared absorption of these materials are outlined.

\section{Current Perspectives in the Science of Quasicrystals}

Since the notion of quasiperiodic order is a very fundamental one, it is reasonable to expect it could be fruitfully applied to quite different sorts of matter arrangements beyond those considered in the previous sections. Some promising advances have been reported during the last decade regarding soft matter structures [54], polymer materials [55,56], or optical lattices in which several monochromatic electromagnetic waves are combined to create an interference pattern exhibiting certain long-range ordering. Then, such a light field is used to trap small particles or atoms within it. Inside the resulting structure the atoms adopt a regular arrangement in space, but they are weakly coupled among them, 
at variance with the usual situation in conventional solid matter. In fact, in atomic condensates based on optical lattices the role of both defects and phonons can be substantially reduced, offering a powerful tool for investigating the quantum behaviour of condensed matter systems under unique control possibilities. Indeed, the symmetry of the optical potential created by the interference of lasers is completely determined by the geometric arrangement of their beams. Therefore, one can in principle design any sort of aperiodic optical potential at will $[57,58]$. Following this approach, Nicolas Macé, Anuradha Jagannathan, and Michel Duneau describe in their contribution a way to obtain a two-dimensional quasiperiodic tiling with eight-fold symmetry using cold atoms [59]. One can obtain a full series of such optical tilings, related by scale transformations, for a series of specific values of the chemical potential of the atoms. A theoretical model for the resulting optical system is described and compared with that of the well-known cut-and-project method for the Ammann-Beenker tiling. On the basis of the obtained results, one expects that this type of cold atom structure may allow for simulation experiments of quantum particles in lattice models with tunable quasiperiodic parameters.

In light of the results reported in the articles collected in this issue, the reader may have arrived to her/his own conclusion regarding where we are going by following QCs' scientific progress. Let me now conclude by adding my own two cents.

It is interesting to note that icosahedral symmetry in condensed matter was first reported in the study of the geometry of certain virus capsids from X-ray diffraction studies of condensed virus particles, that is, virus crystals. Indeed, arguments supporting the presence of a high symmetry degree in virus capsids were put forward early on by F H C Crick and J D Watson, who in 1956 suggested that most of them could be built up from a relatively small number of protein subunits arranged in a symmetrical way, and noticed that of all the possible types of symmetry arrangements only the cubic point groups were likely to lead to an isometric structure [60]. The first experimental evidence for icosahedral symmetry in a virus came from the X-ray diffraction studies of D L D Caspar on tomato bushy stunt virus [61], soon followed by those of A Klug, J T Finch, and R E Franklin on turnip yellow mosaic virus [62]. These investigations confirmed that the $X$-ray diffraction patterns from certain virus crystals showed intensity distributions characteristic of an icosahedral particle. Shortly thereafter, electron microscope observations showed that a number of viruses also had the shape of a regular icosahedron [63]. Subsequently, virus capsids exhibiting the overall shape of a regular dodecahedron were reported as well. Therefore, neither the presence of icosahedral symmetry (first observed in virus assemblies), nor the scale invariance symmetry (also present in fractal structures introduced in the early 1980s [64]) is specific of QCs. However, the combination of both types of symmetries in the same piece of matter is. Accordingly, is it possible that an ensemble of icosahedral virus particles may eventually pile up to give rise to an organic QC? To the best of my knowledge, such a possibility has not yet been fully explored, albeit the use of aperiodic tiling notions, originally introduced in the study of quasicrystalline alloys, has been successfully borrowed to properly classify the protein structure of certain virus families during the last few years [65-67].

Another promising field where the science of quasicrystals may prove useful is number theory. In fact, as soon as one realizes that the regular structure of a perfect lattice is suitable for comparison with regularities among natural numbers, the way is paved for a fruitful relationship between mathematics, crystallography, and physics [68]. In particular, it has been recently conjectured that the distribution of the Riemann Zeta function zeros along the so-called critical line in the complex plane (a long-standing problem known as the Riemann hypothesis and included in the celebrated Hilbert's problems list) may provide a novel example of a one-dimensional quasicrystal structure [69]. Keeping in mind that the solution of the Riemann hypothesis is rewarded with a $\$ 1,000,000$ prize [70], it may occur that keeping up with the pursuit of QCs research could eventually make us not only wiser but a little bit wealthier as well.

After closing the special issue, a new collection "Structure and Properties of Quasicrystals" (http:/ / www.mdpi.com/journal/crystals/special_issues/structure_quasicrystals) is opened. This Collection aims to promote international exchange and to share the latest knowledge and developments in both 
experimental and fundamental aspects in order to gain a deeper understanding on the relationship between the underlying structural order and the resulting physical properties in quasicrystals and their related approximant phases. The capability of exploiting aperiodic order in the design of novel devices based on dielectric multilayers or semiconductor heterostructures is also addressed. Interdisciplinary approaches encompassing the notion of quasiperiodic order in mineralogy, quantum chemistry and bio-inspired systems of current interest will be considered as well.

Acknowledgments: I am greatly indebted to Esther Belin-Ferré, Jean-Marie Dubois, and Uichiro Mizutani for their advice and continued support of my research on quasicrystals and aperiodic structures during the last two decades. I also heartfully thank Luca Dal Negro for his helpful collaboration and interest in topics related to the fundamental aspects of aperiodic order in photonic devices. Their contributions to the field have been a nice source of inspiration to my own work. I warmly thank M. Victoria Hernández for a critical reading of the manuscript and Huimin Lin for her kind editorial assistance during the preparation of this Special Issue.

Conflicts of Interest: The author declares no conflict of interest.

\section{References}

1. Shechtman, D.; Blech, I.; Gratias, D.; Cahn, J.W. Metallic phase with long-range orientational order and no translation symmetry. Phys. Rev. Lett. 1984, 53, 1951-1954. [CrossRef]

2. Levine, D.; Steinhardt, P.J. Quasicrystals: A new class of ordered structures. Phys. Rev. Lett. 1984, 53, 2477-2480. [CrossRef]

3. Steurer, W.; Deloudi, S. Crystallography of Quasicrystals-Concepts, Methods and Structures; Springer Series in Materials Science 126; Springer: Berlin, Germany, 2009.

4. Janssen, T.; Chapuis, G.; de Boissieu, M. Aperiodic Crystals. From Modulated Phases to Quasicrystals; Oxford University Press: Oxford, UK, 2007.

5. IUCr Commission. Report of the Executive Committee for 1991. Acta Cryst. A 1992, 48, 922, The interested reader can find updated information on the crystal definition on IUCr website. Available online: http: //www.iucr.ac.uk/iucr-top/comm/capd/terms.html (accessed on 23 February 2017).

6. Bindi, L.; Steinhardt, P.J.; Yao, N.; Lu, P.J. Natural quasicrystals. Science 2009, 234, 1306-1309. [CrossRef] [PubMed]

7. Bindi, L.; Steinhardt, P.J.; Yao, N.; Lu, P.J. Icosahedrite, Al63Cu24Fe13, the first natural quasicrystal. Am. Mineral. 2011, 96, 928-931. [CrossRef]

8. Bindi, L.; Yao, N.; Lin, C.; Hollister, L.S.; Andronicos, C.L.; Distler, V.V.; Eddy, M.P.; Kostin, A.; Kryachko, V.; MacPherson, G.J.; et al. Natural quasicrystal with decagonal symmetry. Sci. Rep. 2015, 5, 9111. [CrossRef] [PubMed]

9. Bindi, L.; Yao, N.; Lin, C.; Hollister, L.S.; Andronicos, C.L.; Distler, V.V.; Eddy, M.P.; Kostin, A.; Kryachko, V.; MacPherson, G.J. Decagonite, $\mathrm{Al}_{71} \mathrm{Ni}_{24} \mathrm{Fe}_{5}$, a quasicrystal with decagonal symmetry from the Khatyrka CV3 carbonaceous chondrite. Am. Mineral. 2015, 100, 2340-2343. [CrossRef]

10. Boisen, M.B., Jr.; Gibbs, G.V. Mathematical Crystallography. In Reviews in Mineralogy; Ribbe, P.H., Ed.; Mineralogical Society of America: Chantilly, VA, USA, 1985; Volume 15, pp. 191-194.

11. Gévay, G.; Szederkény, T. Quasicrystals and their spontaneous formation possibilities in the nature. Acta Miner. Petrogr. 1988, 29, 5-12.

12. Asimow, P.D.; Lin, C.; Bindi, L.; Ma, C.; Tschauner, O.; Hollister, L.S.; Steinhard, P.J. Shock synthesis of quasicrystals with implications for their origin in asteroid collisions. Proc. Natl. Acad. Sci. USA 2016, 113, 7077-7081. [CrossRef] [PubMed]

13. Pina, C.M.; Sánchez-Acevedo, V. Quasicrystals and other aperiodic structures in mineralogy. Crystals 2016, 6, 137. [CrossRef]

14. Maciá-Barber, E. Thermoelectric Materials: Advances and Applications; Pan Stanford Publishing Pte. Ltd.: Singapore, 2015.

15. Li, R.; Li, Z.; Dong, Z.; Khor, K.A. A review of transmission electron microscopy of quasicrystals-How are atoms arranged? Crystals 2016, 6, 105. [CrossRef]

16. Steurer, W.; Deloudi, S. Decagonal quasicrystals-What has been achieved? C. R. Phys. 2014, 15, 40-47. [CrossRef]

17. De Boissieu, M. Atomic structure of quasicrystals. Struct. Chem. 2012, 23, 965-976. [CrossRef] 
18. Strzalka, R.; Buganski, I.; Wolny, J. Statistical approach to diffraction of periodic and non-periodic crystals-Review. Crystals 2016, 6, 104. [CrossRef]

19. Maciá, E. The role of aperiodic order in science and technology. Rep. Prog. Phys. 2006, 69, 397-441. [CrossRef]

20. Maciá-Barber, E. Aperiodic Structures in Condensed Matter: Fundamentals and Applications; CRC Press: Boca Raton, FL, USA, 2009.

21. Niizeki, K.; Fujita, N. Superquasicrystals: Self-similar-ordered structures with non-crystallographic point symmetries. J. Phys. A Math. Gen. 2005, 38, L199-L204. [CrossRef]

22. Namin, F.A.; Werner, D.H. An exact method to determine the photonic resonances of quasicrystals based on discrete Fourier harmonics of higher-dimensional atomic surfaces. Crystals 2016, 6, 93. [CrossRef]

23. Gaponenko, S.V. Introduction to Nanophotonics; Cambridge University Press: Cambridge, UK, 2010.

24. Yablonovitch, E. Inhibited spontaneous emission in solid-state physics and electronics. Phys. Rev. Lett. 1987, 58, 2059-2062. [CrossRef] [PubMed]

25. Joannopoulos, J.D.; Meade, R.D.; Winn, J.N. Photonic Crystals: Molding the Flow of Light; Princeton University Press: Princeton, NJ, USA, 1995.

26. Maciá, E. Exploiting aperiodic designs in nanophotonic devices. Rep. Prog. Phys. 2012, 75, 036502. [CrossRef] [PubMed]

27. Dal Negro, L.; Lawrence, J.; Trevine, J.; Walsh, G. Aperiodic order for nanophotonics. In Optics of Aperiodic Structures: Fundamentals and Device Applications; Dal Negro, L., Ed.; Pan Stanford Publishing Pte. Ltd.: Singapore, 2014; pp. 1-55.

28. Dal Negro, L.; Wang, R.; Pinhero, F.A. Structural and spectral properties of deterministic aperiodic optical structures. Crystals 2016, 6, 161. [CrossRef]

29. Zito, G.; Rusciano, G.; Sasso, A.; De Nicola, S. Symmetry-induced light confinement in a photonic quasicrystal-based mirrorless cavity. Crystals 2016, 6, 111. [CrossRef]

30. Lifshitz, R. Quasicrystals: A matter of definition. Found. Phys. 2003, 33, 1703-1711. [CrossRef]

31. Elcoro, L.; Pérez-Mato, J.M. Cubic superspace symmetry and inflation rules in metastable MgAl alloy. Eur. Phys. J. B 1999, 7, 85-89. [CrossRef]

32. Dubost, B.; Lang, J.M.; Tanaka, M.; Sainfort, P.; Audier, M. Large AlCuLi single quasicrystals with triacontahedral solidification morphology. Nature (London) 1986, 324, 48-50. [CrossRef]

33. Ohashi, W.; Spaepen, F. Stable GaMgZn quasi-periodic crystals with pentagonal dodecahedral solidification morphology. Nature (London) 1987, 330, 555-556. [CrossRef]

34. Tsai, A.P.; Inoue, A.; Masumoto, T. A stable quasicrystal in Al-Cu-Fe system. Jpn. J. Appl. Phys. 1987, 26, L1505-L1507. [CrossRef]

35. Tsai, A.P.; Yokoyama, Y.; Inoue, A.; Masumoto, T. Quasicrystals in Al-Pd-TM (TM = transition metal) systems prepared by rapid solidification. Jpn. J. Appl. Phys. 1990, 29, L1161-L1164. [CrossRef]

36. Tsai, A.P.; Niikura, A.; Inoue, A.; Masumoto, T.; Nishida, Y.; Tsuda, K.; Tanaka, M. Highly ordered structure of icosahedral quasicrystals in Zn-Mg-RE (RE = rare earth metals) systems. Philos. Mag. Lett. 1994, 70, 169-175. [CrossRef]

37. Tsai, A.P.; Inoue, A.; Masumoto, T. A Stable Decagonal Quasicrystal in the Al-Cu-Co System. Mater. Trans. 1989, 30, 300-304. [CrossRef]

38. Tsai, A.P.; Inoue, A.; Masumoto, T. Stable Decagonal Al-Co-Ni and Al-Co-Cu Quasicrystals. Mater. Trans. 1989, 30, 463-473. [CrossRef]

39. Zhang, X.; Stroud, R.M.; Libbert, J.L.; Kelton, K.F. The icosahedral and related crystal approximant phases in Ti-Zr-Ni alloys. Philos. Mag. B 1994, 70, 927-950. [CrossRef]

40. Tsai, A.P. Discovery of stable icosahedral quasicrystals: Progress in understanding structure and properties. Chem. Soc. Rev. 2013, 42, 5352-5365. [CrossRef] [PubMed]

41. Tsai, A.P.; Guo, J.Q.; Abe, E.; Takahura, H.; Sato, T.J. A stable binary quasicrystal. Nature (London) 2000, 408, 537-538. [CrossRef] [PubMed]

42. Dubois, J.M. Useful Quasicrsytals; World Scientific: Singapore, 2005.

43. Stadnik, Z.M. (Ed.) Physical Properties of Quasicrystals; Springer Series in Solid-State Sciences 126; Springer: Berlin, Germany, 1999.

44. Prekul, A.; Shchegolikhina, N. Two-level electron excitations and distinctive physical properties of Al-Cu-Fe quasicrystals. Crystals 2016, 6, 119. [CrossRef]

45. Li, W.; Fan, T. Elasto-dynamics of quasicrystals. Crystals 2016, 6, 152. [CrossRef] 
46. Hosseini, M.; Sladek, J.; Sladek, V. Elastodynamic analysis of a hollow cylinder with decagonal quasicrystal properties: Meshkess implementation of local integral equations. Crystals 2016, 6, 94. [CrossRef]

47. Kirihara, K.; Nagata, T.; Kimura, K.; Kato, K.; Takata, M.; Nishibori, E.; Sakata, M. Covalent bonds and their crucial effects in pseudogap formation in $\alpha-\mathrm{Al}(\mathrm{Mn}, \mathrm{Re}) \mathrm{Si}$ icosahedral quasicrystalline approximant. Phys. Rev. B 2003, 68, 014205. [CrossRef]

48. Krajčí, M.; Hafner, J. Covalent bonding and bandgap formation in intermetallic compounds: A case study for $\mathrm{Al}_{3}$ V. J. Phys. Condens. Matter 2002, 14, 1865-1880. [CrossRef]

49. Trambly de Laissardière, G.; Nguyen-Manh, D.; Mayou, D. Effective medium-range Mn-Mn pair interaction induces pseudogap in the density of states of $\mathrm{Al}(\mathrm{Si})-\mathrm{Mn}$ approximants. J. Non-Cryst. Solids 2004, 334, 347-351. [CrossRef]

50. Takagiwa, Y.; Kirihara, K. Metallic-covalent bonding conversion and thermoelectric properties of Al-based icosahedral quasicrystals and approximants. Sci. Technol. Adv. Mater. 2014, 15, 044802. [CrossRef] [PubMed]

51. Mizutani, U.; Sato, H. The physics of the Hume-Rothery electron concentration rule. Crystals $2017,7,9$. [CrossRef]

52. Mizutani, U. Hume-Rothery Rules for Structurally Complex Alloy Phases; CRC Press: Boca Raton, FL, USA, 2011.

53. Belin-Ferré, E.; Trambly de Laissardière, G.; Dubois, J.M. Complexity and electronic structure of intermetallics forming push-pull alloys. Crystals 2017, 7, under preparation.

54. Dotera, T. Quasicrystals in soft matter. Isr. J. Chem. 2011, 51, 1197-1205. [CrossRef]

55. Ungar, D.; Percec, V.; Zeng, X.; Leowanawat, P. Liquid quasicrystals. Isr. J. Chem. 2011, 51, $1206-1215$. [CrossRef]

56. Reinhardt, A.; Schreck, J.S.; Romano, F.; Doye, J.P.K. Self-assembly of two-dimensional binary quasicrystals: A possible route to a DNA quasicrystal. J. Phys. Condens. Matter 2017, 29, 014006. [CrossRef] [PubMed]

57. Sánchez-Palencia, L.; Santos, L. Bose-Einstein condensates in optical quasicrystal lattices. Phys. Rev. A 2005, 72, 053607. [CrossRef]

58. Singh, K.; Saha, K.; Parameswaran, S.A.; Weld, D.M. Fibonacci optical lattices for tunable quantum quasicrystals. Phys. Rev. A 2015, 92, 063426. [CrossRef]

59. Macé, N.; Jagannathan, A.; Duneau, M. Quantum simulation of a 2D quasicrystal with cold atoms. Crystals 2016, 6, 124. [CrossRef]

60. Crick, F.H.C.; Watson, J.D. The structure of small viruses. Nature (London) 1956, 177, 473-475. [CrossRef]

61. Caspar, D.L.D. Structure of tomato bushy stunt virus. Nature (London) 1956, 177, 476-477. [CrossRef]

62. Klug, A.; Finch, J.T.; Franklin, R.E. The structure of turnip yellow mosaic virus: X-ray diffraction studies. Biochim. Biophys. Acta 1957, 25, 242, (Aaron Klug was awarded the Nobel Prize in Chemistry 1982 "for his development of crystallographic electron microscopy and his structural elucidation of biologically important nucleic acid-protein complexes"). [CrossRef]

63. Wrigley, N.G. An electron microscope study of the structure of Serecesthis iridescent virus. J. Gen. Virol. 1969, 5, 123-134. [CrossRef] [PubMed]

64. Mandelbrot, B.B. The Fractal Geometry of Nature; Freeman: San Francisco, CA, USA, 1982.

65. Twarock, R. A tiling approach to virus capsid assembly explaining a structural puzzle in virology. J. Theor. Biol. 2004, 226, 477-482. [CrossRef] [PubMed]

66. Janner, A. Towards a classification of icosahedral viruses in terms of indexed polyhedra. Acta Cryst. A 2006, 62, 319-330. [CrossRef] [PubMed]

67. Konetsova, O.V.; Rochal, S.B.; Lorman, V.L. Chiral quasicrystalline order and dodecahedral geometry in exceptional families of viruses. Phys. Rev. Lett. 2012, 108, 038102. [CrossRef] [PubMed]

68. Schumayer, D.; Hutchinson, D.A.W. Colloquium: Physics of the Riemann hypothesis. Rev. Mod. Phys. 2011, 83, 307-330. [CrossRef]

69. Dyson, F. On birds and frogs. Not. Am. Math. Soc. 2009, 56, 212-223.

70. The interested reader can learn more about the Millenium Prize Problems, stated by the Clay Mathematics Institute in 2000. Available online: http:/ / www.claymath.org/ (accessed on 7 February 2017).

(C) 2017 by the author. Licensee MDPI, Basel, Switzerland. This article is an open access article distributed under the terms and conditions of the Creative Commons Attribution (CC BY) license (http:/ / creativecommons.org/licenses/by/4.0/). 